\title{
Effect of Spiritual Intelligence Training on the Anxiety of Medical Sciences Students; a Randomized Clinical Trial Study
}

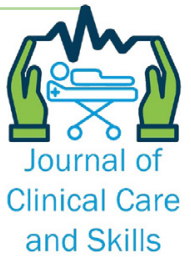

\section{ARTICLE INFO}

\section{Article Type}

Original Research

\section{Authors}

Khoshdeli Z. ${ }^{1} M S C$, Malekzadeh M. ${ }^{2} P h D$ Nooryan $\mathrm{Kh}^{3}{ }^{3} \mathrm{PhD}$ Afroughi $S^{4} P h D$, Zoladl M.*5 $P h D$

How to cite this article
Khoshdeli Z, Malekzadeh M, Noor-
yan Kh, Afroughi S, Zoladl M. Effect
of Spiritual Intelligence Training on
the Anxiety of Medical Sciences Stu-
dents; a Randomized Clinical Trial
Study. Journal of Clinical Care and
Skills. 2020;1(3):115-120.

${ }^{1}$ Student Research Committee, Yasuj University of Medical Sciences, Yasuj, Iran ${ }^{2}$ Psychology Department, Medicine Faculty, Yasuj University of Medical Sciences, Yasuj, Iran

${ }^{3}$ Psychiatric Nursing Department, Nursing Faculty, Yasuj University of Medical Sciences, Yasuj, Iran

${ }^{4}$ Biostatistics \& Epidemiology Department, Health Faculty, Yasuj University of Medical Sciences, Yasuj, Iran

${ }^{5}$ Social Determinants of Health Research Center, Yasuj University of Medical Sciences, Yasuj, Iran

\section{*Correspondence}

Address: Psychiatric Nursing Department, Nursing Faculty, Yasuj University of Medical Sciences, Dr. Jalil Street, Yasuj, Iran. Postal Code: 7591994799. Phone: +98 (74) 33235144 Fax: +98 (74) 33235144 zoladl.mohammad@yums.ac.ir

\section{Article History}

Received: September 15, 2019

Accepted:October 22, 2019

ePublished: September 21, 2020

\begin{abstract}
A B S T R A C T
Aims Anxiety among medical students is a major psychological problem and in the newest meta-analysis, its global prevalence rate is estimated to be $33.8 \%$. Accordingly and since spiritual intelligence training can affect to resolve fundamental issues and enhance individuals' adaptability, therefore the aim of this study was to determine the effect of spiritual intelligence training on the anxiety of medical sciences students.

Materials \& Methods In this randomized clinical trial in 2016, 50 students from Yasuj and Larestan Universities of medical sciences-Iran were selected using purposive sampling method and divided into intervention and control groups with the equal members through random block allocation of 4 . While the control group did not receive any intervention, the spiritual intelligence training accomplished for the intervention group. At the beginning of the study and one month after intervention, the trait and state anxiety of students were measured using Spielberger state-trait anxiety inventory. Data were analyzed using SPSS 21 software, through Chi-square test, independent t-test, and paired t-test.

Findings At the beginning of the study, there was no significant difference between the intervention and control groups regarding the amount of the trait and state anxiety $(p>0.05)$. One month after the intervention, the amount of the trait and state anxiety in the intervention group was significantly less than these amounts in the control group, as well compared to these amounts at the beginning of the study $(\mathrm{p}<0.05)$.

Conclusion The spiritual intelligence training causes a decrease in the trait and state anxiety of medical sciences students.
\end{abstract}

Keywords Spiritual Therapies; Anxiety; Students, Health Occupations

\section{CIT A T ION LINKS}

[1] Role of different neurotransmitters in anxiety ... [2] Diagnostic and statistical manual of mental disorders ... [3] Effectiveness of hypnotherapy on anxiety ... [4] An inventory for measuring clinical anxiety ... [5] The relationship between forgiveness and degrees ... [6] Study of the relationship between problem solving style ... [7] Addressing the mental health concerns of ... [8] Mental health of medical students: a cross-sectional ... [9] Mental health status of the students in Ardabil ... [10] The mental health needs of today's college ... [11] Prevalence of stress among medical students in ... [12] Cigarette smoking: implications for psychiatric ... [13] Alternative projections of mortality and disability by ... [14] Stress, anxiety, depression, and loneliness of graduate... [15] Emotional disorders among Turkish undergraduate ... [16] Changes in mental health status and sources of stress in dental ... [17] Basic nursing: a critical thinking ... [18] Test-taking anxiety among nursing \& general college ... [19] The global prevalence of anxiety among medical ... [20] Psychological stress among undergraduate ... [21] Medical-surgical nursing across the health care ... [22] Spirituality and intelligence: problems and ... [23] Development and determination of content and face ... [24] Spiritual intelligence: the ultimate ... [25] A comparison of spiritual intelligence and emotional ... [26] Principles of spiritual ... [27] Spiritual intelligence: Developing ... [28] Development of the Spanish edition of the ... [29] Psychometric properties of the STAI: a reply ... [30] The effect of Jyoti meditation on student ... [31] The effect of Jyoti meditation on student ... [32] Effectiveness of Training of Spiritual Intelligence ... [33] Spirituality, identity and mental health ... [34] Spiritual care and psychiatric treatment: an ... [35] Rethinking claims of spiritual intelligence: a definition ... 


\section{Introduction}

The term "anxiety" originates from the Latin word "anxieta" meaning mental harassment in some unknown events, and a Greek term "anxo" meaning stress and suffocation. Anxiety is a term used to describe the experiences of ordinary people when faced with threat, danger or stress, and is manifested by severe negative emotions and symptoms, like physical stress in as physical, cognitive, and behavioral changes [1-3].

The abnormal functioning of GABA neurotransmitters, serotonin, norepinephrine and dopamine, as well as the abnormal response of chemical receptors lead to anxiety, in which a person with anxiety predicts a danger or misery in future. This condition may indirectly as trait anxiety or directly as state anxiety affects people's lives and causes psychological and social changes, such as doubts, inability to make decisions, sensitivity, anger, loneliness, helplessness, and hatred [1, 4].

Trait anxiety is associated with the potential for anxiety, through which individuals are prepared to perceive anxious situations, such as experiencing danger or threatening situations, and in response to such situations, severely reveal anxious reactions; whereas state anxiety refers to the tangible and visible response to anxiety at certain times and severely inappropriate situations, such as at discussions, loss of social status or threatened human security and health [5].

Students, as the most important human capital, are the most influential factors to form the nature of each society and the cultural, social and economic development that their mental health, including selfacceptance, positive relationship with others, independence, environmentally dominant, purposefulness in life, and personal growth, in addition to positive personal effects, play an important role in the dynamics and efficiency of societies [6-8].

Despite the importance of students' mental health, previous studies have suggested that entering university as an environment different from school environment and facing new situations poses problems, such as stress and anxiety for students and jeopardizes their mental health [9-11].

Evolution-based crises associated with adolescence, being far from the family, fear of self-expression while facing with others, a desire for show-off, being exposed with the opposite sex, living with other students in the dormitory, lack of facilities, financial problems, unfamiliar environments, dissatisfaction with the major, academic problems, competition with other students, tests and several classes to attend, and being worried about job prospects are factors that increase trait anxiety and state anxiety in students [12-16].

Anxiety has always been seen among medical students due to the overwhelming nature of these disciplines and the necessity of practices based on theoretical education in the patient's bedside and in the latest meta-analysis, the prevalence of this psychological disorder in medical students is estimated $33.8 \%$ worldwide. Accordingly, appropriate interventions at three levels of prevention are important to control medical students' anxiety [11, 17-20].

Drug therapy as a routine intervention can be effective in improving anxiety and its symptoms by balancing biochemical neurochemical mediators. However, since other psychological pathological processes, such as decreased levels of psychological immunity, unconscious effects, pathophysiological changes originating from the environment, the individuals' attitudes toward events and their supportive system have also been effective in causing anxiety and their interventions have less complications than drug interventions, therefore, nowadays non-pharmacological therapies have been considered in anxiety-related interventions [3, 21].

One of the non-pharmacological interventions regarding anxiety is spiritual intelligence training. Spiritual intelligence is a profound intuition that makes a person with more self-conscious about the dimensions of himself, inner knowledge based on good understanding and unity with the universe and all living on it and also the ability to use sacred elements, finding meaning in life, high vigilance and transcendence, use an adaptive approach that leads to the production of valuable results. Accordingly, spiritual intelligence training provides the means for a person to use spiritual information in a consistent manner to solve life's problems and finding meaning in life and values to achieve his goals [22, 23].

Considering the above issues and regarding the fact that spiritual intelligence training by facilitating the communication with a superior sacred power, developing beliefs, norms, and values related to this superior power and cultivating spiritual capacities and abilities can make life meaningful and valuable and help the individual solve basic problems and increase adaptability [24-27], this study aimed at determining the effect of spiritual intelligence training on anxiety of medical students.

\section{Materials and Methods}

This study is a randomized clinical trial that was conducted in the Yasuj and Larestan Universities of Medical Sciences, Iran in 2016. The purposive sampling method was done and 424 students of the Yasuj and Larestan Universities were selected. After explaining the research objectives for the students, the consent form and the research tools were distributed among them. Of these students, 385 cases were willing to participate in the study and completed the written informed consent form. Of those willing to participate in the research, a student who was eligible regarding the inclusion and 
exclusion criteria was allocated to the intervention and control groups through random allocation with a block size of four and continued until the sample size in each group reach 25 subjects (a total of 50 subjects).

Inclusion criteria included obtaining the score of 43 and above (cut-off point) in trait and state anxiety in the Spielberger State-Trait Anxiety Inventory and the exclusion criteria were a diagnosed mental illness or chronic physical illness.

The demographic and background information questionnaire was used to collect characteristics, such as age, sex, marital status, field of study, university entry year, university name, place of residence, history of diagnosed chronic physical and mental diseases and also the Spielberger State-Trait Anxiety Inventory was used as research tools.

Spielberger State-Trait Anxiety Inventory: The 40-item State-Trait Anxiety Inventory was developed in 1971 by Spielberger. It takes about 6 minutes to complete this self-administrated checklist. This inventory consists of two parts of 20 questions for measuring hidden anxiety (trait anxiety) and 20 questions for measuring apparent anxiety (state anxiety) with different instructions for completing each section, therefore, the examiner emphasized to the subjects that they should study both instructions carefully and choose the most appropriate option.

According to the test protocol, the subjects in 20 questions regarding trait anxiety based on their usual and frequent feelings should choose an option from "almost never", "sometimes", "most often" and "almost always" that are scored 1 to 4 . It is worth noting that the questions $21,23,26,27,30,33,34$, 36 and 39 are reversely scored and the answers are scored 4 to 1 , respectively.

Also, according to its protocol, the subjects should answer 20 questions regarding state anxiety based on their own emotions at the time of completing the form. They should choose an option that best expresses their intensity of feeling on a four-choice scale "very low", "low", "many" and "too many". The answers are scored 1 to 4 . It is worth noting that the questions $1,2,5,8,10,11,15,16,19$ and 20 are reversely scored and the answers are scored 4 to 1 , respectively.

To obtain the score of each type of anxiety, the total score of the questions is calculated, and higher scores indicate more anxiety in the respondent. The range of trait and state anxiety varied between 20 and 80 , with a score of 43 being suggested as the cut-off point. Also, according to the scores obtained for each type of anxiety, the trait anxiety (hidden anxiety) and situational anxiety (apparent anxiety) can be classified (Table 1).

The validity of the Spielberger Inventory was assessed through construct validity and approved and obtained $0.75-0.83$ due to its correlation coefficient compared with other validated anxiety assessment questionnaires, such as the Anxiety Scale Questionnaire (ASQ) and the Taylor's Manifest Anxiety Scale (TMAS). Its validity has also been evaluated and validated in a concurrent validity. The reliability of the Spielberger State-Trait Anxiety Inventory was confirmed in the range of 0.87-0.91 based on the Cronbach's alpha coefficient reported in previous studies [28, 29]. In the present study, Cronbach's alpha coefficient of Spielberger inventory was 0.93 .

The control group did not receive any intervention, whereas the intervention group was subjected to eight 90-minute sessions of spiritual intelligence training, once every 4 days. In training sessions, topics on defining and explaining spiritual intelligence and its components, self-awareness, intelligent awareness, transcendental consciousness, personal meaning, meaning in life, truth, and honesty were presented using lectures, questions, answers, and some practices based on the protocol (Table 2).

Table 1) Classification of the anxiety severity based on the Spielberger State-Trait Anxiety Inventory

\begin{tabular}{|l|cc|}
\hline \multicolumn{1}{c}{ Classification of the anxiety } & $\begin{array}{c}\text { State anxiety } \\
\text { score }\end{array}$ & $\begin{array}{c}\text { Trait anxiety } \\
\text { scores }\end{array}$ \\
\hline Mild anxiety & $20-31$ & $20-31$ \\
\hline Moderate to low anxiety & $32-42$ & $32-42$ \\
Moderate to high anxiety & $43-53$ & $43-52$ \\
Relatively severe anxiety & $54-64$ & $53-62$ \\
Sever anxiety & $65-75$ & $63-72$ \\
Very severe anxiety & $76-80$ & $73-80$ \\
\hline
\end{tabular}

Table 2) The content of the spiritual intelligence training sessions

\section{First session}

Introduction, expressing expectations and rules, defining and explaining spiritual intelligence and its components, defining consciousness and self-awareness, and entering spiritual states while being conscious, training awareness of your own anxiety response

Second session

Explaining the effects of spiritual intelligence on daily life and interactions, training existential concepts (reality, death), training mental imagery, recognizing your strengths and weaknesses

Third session

Describing phobias-related thoughts and behaviors, supporting patients in coping with long-avoided anxieties, critical thinking training

Fourth session

Personal meaningfulness, training different coping styles, ability and training making a purposeful life and the material and physical experiences, ability to adorn daily activities with a sense of spirituality and holiness

Fifth session

Meaning of life, experiencing meaning and communicating between life's valuable activities and experiences, revisiting your world with an emphasis on the meaning of life

Sixth session

Familiarity with the visualization and vision concepts, transcendent consciousness and mindfulness training, holism and understanding and identification of the dimensions and capabilities of yourself, others, and the world

Seventh session

Familiarity with the concepts, including truth, honesty, selfacceptance, accepting others, virtuous behaviors, such as forgiveness, remission, gratitude, positive thinking and optimism, anger control skills training, daring, positive interpersonal relationships, and stress control skills

Eighth session

Familiarity with the holiness, purity and superiority, alliance, unity and integrity, spiritual education, development of spiritual awareness 
One month after the end of the intervention, the Spielberger inventory was again completed by all subjects and the collected data were analyzed by SPSS 21 at 95\% confidence level. Inter-group comparison of the demographic quantitative variables, including age and the dependent variables, including trait and state anxiety with normal distribution was performed using independent t-test. Chi-square test was used to compare the qualitative variables, including demographic and background variables, such as gender, marital status, field of study, year of entry to university, university name, and place of residence. Paired t-test was used for intra-group comparison of the dependent variables, including trait and state anxiety with normal distribution.

All ethical considerations, such as obtaining the written informed consent from the students, making the subjects informed about the possibility to withdraw from the study, confidentiality of the information and general use of data based on the study objectives were observed. Also, considering the positive effects of spiritual intelligence training on trait and state anxiety of the intervention group, after preparing the final report of the research plan, spiritual intelligence training was conducted for the control group according to the protocol.

\section{Findings}

The mean age of the students was $22.04 \pm 2.56$ years. The mean age of the intervention and control groups were $22.56 \pm 3.32$ and $21.52 \pm 1.36$ years, respectively. There was no significant difference in age at baseline between the study groups ( $p>0.05)$.

The intervention and control groups were homogenized at baseline in terms of demographic and background variables, such as gender, marital status, field of study, the year attending to the university, the university and place of residence and there was no significant difference between them ( $>0.05$; Table 3).

The level of trait and state anxiety was similar in the medical students in the intervention and control groups at the baseline $(p>0.05)$. One month after intervention, trait and state anxiety were significantly lower in the intervention group compared to the control group $(\mathrm{p}<0.05)$.

In the intra-group comparison, considering the difference between the mean scores of trait anxiety and state anxiety in the intervention group ($0.98 \pm 7.96$ and $-12.76 \pm 9.01$, respectively) and the control group $(-1.80 \pm 8.59$ and $-1.72 \pm 9.33$, respectively), the trait anxiety and state anxiety of the intervention group decreased significantly after the intervention $(p<0.05)$; however, there was no significant difference in the control group after the intervention compared to the baseline $(p>0.05$; Table 4).
Table 3) Comparison of the absolute and relative frequency distribution of demographic and background qualitative variables of medical students in intervention and control groups at baseline $(n=25$ per group; the numbers in parentheses are percent)

\begin{tabular}{|c|c|c|c|}
\hline Variables & $\begin{array}{c}\text { Interventio } \\
\text { n group }\end{array}$ & $\begin{array}{l}\text { Control } \\
\text { group }\end{array}$ & P. value \\
\hline \multicolumn{4}{|l|}{ Gender } \\
\hline Male & $12(48.0)$ & $7(28.0)$ & \multirow{2}{*}{0.15} \\
\hline Female & $13(52.0)$ & $18(72.0)$ & \\
\hline \multicolumn{4}{|l|}{ Marital status } \\
\hline Single & $23(92.0)$ & $24(96.0)$ & \multirow{2}{*}{0.55} \\
\hline Married & $2(8.0)$ & $1(4.0)$ & \\
\hline \multicolumn{4}{|l|}{ Major } \\
\hline Medicine/ Dentistry & $2(8.0)$ & $5(20.0)$ & \multirow{4}{*}{0.32} \\
\hline $\begin{array}{l}\text { Nursing/Midwifery/ Medical } \\
\text { emergencies }\end{array}$ & $18(72.0)$ & $12(48.0)$ & \\
\hline $\begin{array}{l}\text { Laboratory sciences/ Operating } \\
\text { room/ Anesthesia }\end{array}$ & $3(12.0)$ & $6(24.0)$ & \\
\hline $\begin{array}{l}\text { Public Health/ Environmental } \\
\text { Health/ Nutrition }\end{array}$ & $2(8.0)$ & $2(8.0)$ & \\
\hline \multicolumn{4}{|l|}{ Year of University entrance } \\
\hline 2011 & 0 & $1(4.0)$ & \multirow{5}{*}{0.65} \\
\hline 2012 & $7(28.0)$ & $4(16.0)$ & \\
\hline 2013 & $2(8.0)$ & $4(16.0)$ & \\
\hline 2014 & $7(28.0)$ & $7(28.0)$ & \\
\hline 2015 & $9(36.0)$ & $9(36.0)$ & \\
\hline \multicolumn{4}{|l|}{ University } \\
\hline $\begin{array}{l}\text { Yasuj University of Medical } \\
\text { Sciences }\end{array}$ & $11(44.0)$ & $12(48.0)$ & \multirow{2}{*}{0.78} \\
\hline $\begin{array}{l}\text { Lorestan School of Medical } \\
\text { Sciences }\end{array}$ & $14(56.0)$ & $13(52.0)$ & \\
\hline \multicolumn{4}{|l|}{ Living status } \\
\hline Dormitory & $21(84.0)$ & $22(88.0)$ & \multirow{2}{*}{0.68} \\
\hline At home & $4(16.0)$ & $3(12.0)$ & \\
\hline
\end{tabular}

Table 4) Comparison of the mean scores of trait and state anxiety of the medical students at baseline and one month after intervention in the two groups

\begin{tabular}{|c|c|c|c|}
\hline Variables & $\begin{array}{l}\text { Control } \\
\text { group }\end{array}$ & $\begin{array}{c}\text { Intervention } \\
\text { group }\end{array}$ & $\begin{array}{c}\text { Inter-group } \\
\text { P. value }\end{array}$ \\
\hline \multicolumn{4}{|l|}{ Trait anxiety } \\
\hline At baseline & $51.08 \pm 6.82$ & $52.0 \pm 8.27$ & 0.67 \\
\hline $\begin{array}{l}\text { One month after } \\
\text { intervention }\end{array}$ & $49.28 \pm 7.47$ & $42.2 \pm 9.42$ & 0.005 \\
\hline Intra-group $\mathrm{P}$ value & 0.31 & 0.0001 & - \\
\hline \multicolumn{4}{|l|}{ State anxiety } \\
\hline At baseline & $53.60 \pm 8.46$ & $54.68 \pm 9.71$ & 0.68 \\
\hline $\begin{array}{l}\text { One month after } \\
\text { intervention }\end{array}$ & $51.88 \pm 6.78$ & $41.92 \pm 8.41$ & 0.0001 \\
\hline Intra-group P value & 0.37 & 0.0001 & - \\
\hline
\end{tabular}

\section{Discussion}

The findings of the present study indicated that the intervention and control groups were homogenized in terms of quantitative and qualitative demographic and contextual variables as well as trait and state anxiety at the baseline. Therefore, the changes in the dependent variables, including trait and state anxiety of the medical students after the intervention were due to the effect of spiritual intelligence training as an independent variable.

The results of inter-group comparison showed that one month after intervention, trait and state anxiety were significantly lower in the intervention group compared to the control group. Also, based on the results of intra-group comparison, the level of trait and state anxiety of the intervention group students decreased significantly one month after the intervention compared to the baseline, whereas in the control group, there was no significant 
difference in trait and state anxiety of the students one month after the study intervention compared to the baseline; therefore, it can be concluded that spiritual intelligence training as a research intervention was able to differentiate between the intervention and control groups and significantly reduced trait and state anxiety in the medical students participating in the intervention group.

These results are consistent with results of Gutierrez [30], Souri et al. [31], and Ganjavi [32] studies. Also, the findings of the present study on the positive effects of spiritual intelligence training on reducing trait and state anxiety in medical students based on theoretical knowledge bases are also rational and justified; because spirituality is a stage, in which meaning, hope, comfort and inner peace are received, and spiritual intelligence represents a set of spiritual abilities and capacities that its application in daily life enhances one's adaptability. Belief in God or a superior power in the spirituality stage, by creating a sense of being supported, creates a sense of mastery over one's condition and positive attitude and reduces trait anxiety. Increasing the meaning, purpose and patience in life caused by increased spiritual intelligence also helps to cope with life's problems, thereby raising the threshold of tolerance and resilience to problems and stresses and reducing the state anxiety [33-35].

In addition to demonstrating the positive therapeutic effects of spiritual intelligence training in reducing trait and state anxiety in medical students in this study, due to the availability, costeffectiveness, effect at shorter time, and minor side effects of this intervention, after confirming the results of this study in other relevant studies, spiritual intelligence training can be used as a complementary therapy to manage and control trait and state anxiety in medical students.

The present study also had some limitations; for example, the possible conversation between the students in the intervention and control groups and the possibility of exchanging information between them regarding educational contents and training could affect the results of the study. The possible reactions of individuals after learning about their anxiety at the baseline and also the involuntary and unconscious tendency to present their anxiety in a favorable range within a month after the end of interventions are other limitations of this study that could affect the results. Although students in the study were initially asked to refrain from attending other educational or psychological intervention courses or obtaining information during their study, however, the lack of attention to this issue by the intervention and control groups could affect the results of the study. Therefore, it is necessary to consider the above limitations to interpret the results of this study.

Considering the mentioned issues and in order to improve the mental health of medical students, it is suggested to take necessary measures at the beginning of their entrance to university through holding workshops on spiritual intelligence training. Also, as medical students experience other negative consequences, such as depression, stress, etc., it is recommend that further studies examine the impact of spiritual intelligence training on these variables in medical students.

\section{Conclusion}

Spiritual intelligence training reduces trait and state anxiety in medical students and as an adjunctive therapy is effective in reducing anxiety and promoting mental health of medical students.

Acknowledgments: This study was extracted from a research approved by the Research Council of Yasuj University of Medical Sciences. The authors are thankful to the staff of the School of Nursing and the Vice-Chancellor for Research and Technology of Yasuj University of Medical Sciences, the students who participated in the study and all those who contributed to this study.

Ethical Permission: This study was approved by the Research Ethics Committee of Yasuj University of Medical Sciences (IR.YUMS.REC.1395.23) and registered at the Iranian clinical trial database (IRCT2016041627410N1).

Conflict of interests: None declared.

Authors' Contribution: Khoshdeli Z. (First author), Introduction author/ Original researcher/ Discussion author (20\%); Malekzadeh M. (Second author), Methodologist/ Assistant (10\%); Nourian K. (Third author), Introduction author/ Assistant (10\%); Afrooghi S. (Fourth author), Assistant/ Statistical analyst (10\%); Zoladl M. (Fifth author), Introduction author/ Methodologist/ Original researcher/ Statistical analyst/ Discussion author (50\%)

Funding: This research has been funded by the Vice Chancellor for Research and Technology of Yasouj University of Medical Sciences.

\section{References}

1- Kaur S, Singh R. Role of different neurotransmitters in anxiety: a systemic review. Int J Pharm Sci Res. 2017;8(2):411-21.

2- American Psychiatric Association. Diagnostic and statistical manual of mental disorders (DSM-5TM). $5^{\text {th }}$ Edition. Washington DC: American Psychiatric Association; 2013.

3- Lotfifar B, Karami A, Sharifi Daramadi P, Fathi M. Effectiveness of hypnotherapy on anxiety level. Res Med. 2013;37(3):164-70. [Persian]

4- Beck AT, Epstein N, Brown G, Steer RA. An inventory for measuring clinical anxiety: psychometric properties. J Consult Clin Psychol. 1988;56(6):893-7.

5- Ghobari Bonab B. The relationship between forgiveness and degrees of anxiety among the parents of execeptional and ordinary children. Howzeh Univ J. 2002;8(31):78-96. [Persian]

6- Babapour Kheyroddin J, Rasoulzadeh Tabatabaei K, Ejei J, Fathi Ashtiani A. study of the relationship between problem solving style and psychological well-being among university students. J Psychol. 2003;7(1):3-16. [Persian] 
7- Mori SC. Addressing the mental health concerns of international students. J Couns Dev. 2000;78(2):137-44.

8- Shariati M, Yunesian M, Vash JH. Mental health of medical students: a cross-sectional study in Tehran. Psychol Rep. 2007;100(2):346-54.

9- Dadkhah B, Mohammadi MA, Mozaffari N. Mental health status of the students in Ardabil university of medical sciences, 2004. J Ardabil Univ Med Sci. 2006;6(1):31-6. [Persian]

10- Kitzrow MA. The mental health needs of today's college students: challenges and recommendations. NASPA J. 2009;46(4):646-60.

11- Sani M, Mahfouz M, Bani I, Alsomily A, Alagi D, Alsomily $\mathrm{N}$, et al. Prevalence of stress among medical students in Jizan University, Kingdom of Saudi Arabia. Gulf Med J. 2012;1(1):19-25.

12- Glassman AH. Cigarette smoking: implications for psychiatric illness. Am J Psychiatry. 1993;150(4):546-53. 13- Murray CJ, Lopez AD. Alternative projections of mortality and disability by cause 1990-2020: global burden of disease study. Lancet. 1997;349(9064):1498504.

14- Byars JL. Stress, anxiety, depression, and loneliness of graduate counseling students: the effectiveness of group counseling and exercise [Dissertation]. Lubbock, TX: Texas Tech University; 2005.

15- Nur N, Cetinkaya S, Sabanciogullari V, Ozsahin SL, Kavakci O. Emotional disorders among Turkish undergraduate medical students. Pakistan J Med Sci. 2008;24(6):792-7.

16- Esfahanizadeh K, Ziaei S, Mirsharif Z, Nasri S, Mirsharif F, Valaei N, et al. Changes in mental health status and sources of stress in dental students of Islamic Azad University, Tehran. Pajoohandeh J. 2013;18(1):34-9. [Persian]

17- Potter PA, Perry AG. Basic nursing: a critical thinking approach. 4th Edition. St. Louis: Mosby; 1999.

18- Brewer T. Test-taking anxiety among nursing \& general college students. J Psychosoc Nurs Ment Health Serv. 2002;40(11):22-9.

19- Quek TT, Tam WW, Tran BX, Zhang M, Zhang Z, Ho CS, et al. The global prevalence of anxiety among medical students: a meta-analysis. Int J Environ Res Public Health. 2019;16(15):E2735.

20- Sherina M, Rampal L, Kaneson N. Psychological stress among undergraduate medical students. Med J Malaysia. 2004;59(2):207-11.

21- Donna D, Ignatavicious M, Workman L, Mishler M.
Medical-surgical nursing across the health care continuum. 3rd Edition. Philadelphia: W.B. Saunders; 1999.

22- Emmons RA. Spirituality and intelligence: problems and prospects. Int J Psychol Relig. 2000;10(1):57-64.

23- Khodayarifard M, Khorami Markani A, Ghobari Bonab

B, Sohrabi F, Zamanpour E, Raghebian R, et al. Development and determination of content and face validity of spiritual intelligence scale in iranian students. Appl Psychol Res Q. 2017;7(4):39-49. [Persian]

24- Zohar D. Spiritual intelligence: the ultimate intelligence. London: Bloomsbury Publishing; 2012.

25- Sobhi Garamaleki N, Porzoor P. A comparison of spiritual intelligence and emotional intelligence of college students and religious students. J Cult Islam Univ. 2014;4(2):297-310.[Persian]

26- Sohrabi F. Principles of spiritual intelligence. J Ment Health. 2008;1(1):14-8. [Persian]

27- Sisk DA. Spiritual intelligence: Developing higher consciousness revisited. Gift Educ Int. 2016;32(3):194208.

28- Spielberger CD, Gonzalez-Reigosa FE, MartinezUrrutia AN, Natalicio L, Natalicio DS. Development of the Spanish edition of the state-trait anxiety inventory. Interam J Psychol. 1971;5(3-4):145-58.

29- Speilberger CD, Vagg PR. Psychometric properties of the STAI: a reply to Ramanaiah, Franzen, and Schill. J Pers Assess. 1984;48(1):95-7.

30-Gutierrez D. The effect of Jyoti meditation on student counselor emotional intelligence, stress, and daily spiritual experiences [Dissertation]. Orlando, Florida: University of Central Florida; 2014.

31- Souri A, Azadmard S, Zahedi O, Ashoori J. The effect of meta-cognitive therapy and educating the components of spiritual intelligence on generalized anxiety disorder of students. Iran J Health Educ Health Promot. 2016;3(4):349-59. [Persian]

32- Ganjavi LMS. Effectiveness of Training of Spiritual Intelligence Components on consequences of psychological and self-esteem of Adolescents. Int J Indian Psychol. 2016;3(3):74-84.

33- Ghamari Givi H. Spirituality, identity and mental health in the life span. Psychol Relig. 2009;1(4):10-9. [Persian]

34- Culliford L. Spiritual care and psychiatric treatment: an introduction. Adv Psychiatr Treat. 2002;8(4):249-58. 35- King DB. Rethinking claims of spiritual intelligence: a definition, model, and measure. ProQuest; 2008. 